\title{
Territorio, paisaje y herencia industrial: debates y acciones en el contexto europeo ${ }^{1}$
}

\author{
Paz Benito del Pozo \\ Universidad de León. Departamento de Geografía y Geología \\ mpbenp@unileon.es
}

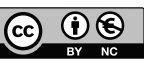

Recepción: octubre de 2011

Aceptación: febrero de 2012

\begin{abstract}
Resumen
Este trabajo se sitúa en las coordenadas del análisis geográfico de los desafíos que plantea la sociedad postindustrial y ofrece una reflexión acerca del patrimonio industrial como recurso y como oportunidad para los espacios afectados por procesos que generan abandono de fábricas y suelos industriales. Desde la óptica de la experiencia acumulada en España, se revisa la evolución del debate teórico sobre "patrimonio industrial, territorio y cultura" y se analizan las acciones europeas tras dos décadas de intervenciones desiguales. Finalmente, se repasan los nuevos enfoques en la interpretación y el uso de la herencia industrial, enfoques en los que el paisaje sustituye al monumento y el territorio cobra protagonismo; un discurso que pone el énfasis en la memoria de los lugares, así como en el componente subjetivo y emocional del patrimonio industrial.
\end{abstract}

Palabras clave: patrimonio industrial; suelos abandonados; memoria del lugar; viejas fábricas; cultura del territorio.

Resum. Territori, paisatge $i$ herència cultural: debats $i$ accions en el context europeu

Aquest treball se situa en les coordenades de l'anàlisi geogràfica dels desafiaments que planteja la societat postindustrial i ofereix una reflexió al voltant del patrimoni industrial com a recurs i com a oportunitat per als espais afectats per processos que generen abandonament de fabriques i sòls industrials. Des de l'òptica de l'experiència acumulada a Espanya, s'hi revisa l'evolució del debat teòric sobre "patrimoni industrial, territori i cultura» i s'hi analitzen les accions europees després de dues dècades d'intervencions desiguals. Finalment, s'hi repassen els nous enfocaments en la interpretació i l'ús de l'herència industrial, enfocaments en què el paisatge substitueix el monument i el territori cobra protagonisme; un discurs que posa l'èmfasi en la memòria dels llocs, així com en el comportament subjectiu i emocional del patrimoni industrial.

Paraules clau: patrimoni industrial; sòls abandonats; memòria del lloc; fabriques velles; cultura del territori.

1. Este trabajo se inscribe en el Proyecto de Investigación $\mathrm{I}+\mathrm{D}+\mathrm{i}$ financiado por el Ministerio de Ciencia e Innovación, Plan Nacional 2008-2011, convocatoria 2010. Referencia: CSO2010-18471. 
Résumé. Territoire, paysage et patrimoine industriel: des débats et des actions dans le contexte européen

Ce travail s'inscrit dans le cadre des coordonnées de l'analyse géographique des défis de la société postindustrielle et propose une réflexion sur le patrimoine industriel comme une ressource et une opportunité pour les zones touchées par les processus qui génèrent des usines désaffectées et des sols industriels. Il examine, du point de vue espagnol, l'évolution du débat théorique sur le "patrimoine industriel, le territoire et la culture» et examine l'expérience européenne, après deux décennies d'interventions inégales. Enfin, nous examinons des nouvelles approches en ce qui concerne l'interprétation et l'utilisation du patrimoine industriel. Le paysage remplace le monument et le territoire entre en scène, un discours qui met l'accent sur la mémoire des lieux ainsi que sur l'élément subjectif émotionnel du patrimoine industriel.

Mots clé: patrimoine industriel; abandon; mémoire du lieu; vieilles usines; culture du territoire.

Abstract. Territory, landscape and industrial heritage: Debates and actions in the European context

This paper falls within the scope of the geographical analysis of the challenges posed by post-industrial society and offers some thoughts on industrial heritage both as a resource and an opportunity for areas affected by processes leading to the abandonment of factories and industrial sites. From a Spanish viewpoint, an overview is given of the developments in the theoretical debate on "industrial heritage, territory and culture" and an analysis is provided of European experiences after two decades of uneven interventions. Finally, a review is offered of new approaches to the interpretation and use of industrial heritage; approaches in which landscapes replace monuments and territory gains a leading role. This is a line of thought which emphasizes local memories together with the subjective and emotional component of industrial heritage.

Keywords: industrial heritage; derelict sites; local memories; old factories; regional culture.

\section{Sumario}

El interés social por las ruinas industriales

Patrimonio industrial, identidad

Programas de conservación y proyecto local: planteamientos a debate y recuperación del patrimonio industrial Conclusiones

Paisajes e itinerarios industriales: Bibliografía nuevos enfoques integradores

\section{El interés social por las ruinas industriales}

Podemos afirmar que en España el interés social por las ruinas industriales (fábricas, explotaciones mineras, barrios obreros, poblados de empresa, etcétera) llega con retraso respecto a los países europeos de tradición industrial y arranca del proceso general de desindustrialización de la década de 1980 y de las políticas para reactivar la economía de los lugares más afectados. 
El cierre de empresas, el despido masivo de trabajadores y la multiplicación de espacios abandonados se contempla, inicialmente, como un problema económico y laboral que mueve a los políticos a buscar soluciones de tipo sectorial: ayudas a las empresas, formación para los trabajadores, creación de infraestructuras industriales, incentivos a la inversión, etcétera. En esta etapa, el territorio no es una prioridad; no existe experiencia sobre la desaparición generalizada de industrias ni sobre el problema de los suelos industriales abandonados a gran escala, su impacto en la ciudad y en los espacios subordinados a la actividad industrial. Parece normal eliminar las viejas fábricas y retener fuera del mercado, con fines especulativos, los terrenos liberados por la industria. La sociedad, en suma, no se plantea la crisis del modelo de ciudad industrial ni percibe la problemática urbanística asociada a las ruinas industriales; como tampoco vislumbra el potencial generador de oportunidades y riqueza de tales ruinas.

Pero cuando los efectos de la crisis industrial y urbana se hacen evidentes, y las políticas de reindustrialización muestran sus limitaciones para crear industrias nuevas en los lugares de industrialización antigua, la mirada se vuelve sobre las ruinas heredadas: ¿qué hacer?, ¿cómo resolver los impactos medioambientales y urbanísticos de las factorías, los talleres y los ferrocarriles abandonados? Surge entonces una sólida corriente de opinión y de pensamiento sensible con las ruinas industriales, los problemas que acarrean y la necesidad de buscar soluciones. Esta preocupación cristaliza en la década de 1990 en propuestas de intervención fundadas en la idea de proteger y conservar las estructuras, los edificios y los espacios industriales abandonados en lugar de suprimir o eliminar las huellas de la vieja industria. Este giro en el modo de plantear las acciones sobre las ruinas industriales se justificó, desde las plataformas prodefensa del patrimonio y algunas instancias académicas, con diferentes argumentos: por ser vestigios del pasado con valor testimonial o elementos de la arqueología industrial; por tratarse de un recurso con valor cultural capaz de actuar como reclamo turístico, y, por último, por ser un poderoso elemento de la memoria y la identidad de los lugares (Forner, 1989; Feliú, 1998; Incuna, 2002).

¿Por qué cambia el enfoque español del problema? La respuesta hay que buscarla en la reacción de los países europeos pioneros en la industrialización, donde la experiencia de la crisis del modelo de producción fordista fue temprana y muy intensa: Reino Unido, Francia, Bélgica y Alemania. En estos países, tras la Segunda Guerra Mundial, los científicos sociales lanzaron las primeras señales de alarma sobre la destrucción de la herencia industrial, cuando la devastación bélica sirvió de excusa para arrasar las viejas industrias e infraestructuras que simbolizaban el progreso en muchas ciudades y territorios. Esta sensibilidad adquirió una dimensión social a partir de lo que hoy se considera un hito: la destrucción, en 1962, de la Euston Station de Londres, que consigue remover conciencias y movilizar a amplios colectivos en favor de la herencia industrial.

En el plano académico y conceptual también se producen aportaciones en esa línea sensible con el legado de la industrialización. Destacan los trabajos pioneros de Hudson $(1963,1979)$ y Buchanan (1972), que proponen interpre- 
tar las ruinas industriales como parte de la herencia cultural y como elementos con valor científico dentro de una nueva disciplina llamada «arqueología industrial». Hudson definió el objeto de la arqueología industrial como el descubrimiento, la catalogación y el estudio de los restos físicos, las infraestructuras de transportes y comunicaciones y la documentación sobre la historia industrial; Buchanan explicó esta ciencia como un campo de estudio práctico-teórico que busca la protección de los monumentos industriales y centra el análisis de los vestigios industriales en el contexto de la historia social y tecnológica. Aunque la arqueología industrial se impuso en los ambientes académicos, en la práctica no dio una respuesta satisfactoria al problema de las ruinas industriales en ningún país europeo (Cossons, 1975; Borsi, 1978; Hudson, 1979; Daumas, 1980; Pinard, 1985). A esa nueva ciencia le faltaba la dimensión aplicada, la capacidad de proponer acciones sobre la herencia industrial tomando en cuenta el territorio y los actores sociales y, sobre todo, le faltaba realismo respecto al desarrollo urbanístico, pues en las ciudades industriales no era posible conservar la vasta extensión de ruinas que el cambio de modelo productivo y de paradigma tecnológico estaban generando.

A lo largo de la década de 1980 y principios de la de 1990 los expertos (sobre todo urbanistas, gestores públicos y arquitectos) abren un nuevo frente de discusión, un debate que trasciende el mero rescate del edificio industrial y plantea una verdadera estrategia territorial sobre el problema de las ruinas industriales. Las preguntas clave son estas: ¿Es racional que la industria invada nuevos terrenos agrícolas y deje sin uso los antiguos suelos abandonados?; ¿existe una lógica inexorable que conduce al despilfarro de edificios y terrenos en vez de propiciar su reutilización?; jes posible considerar los restos más representativos de la industria como parte del patrimonio cultural y promover su protección y conservación? Este último interrogante genera un profundo debate entre autores como Newell, Negri, Bertelli, Selvafolta y Bisi, cuyas ideas se divulgaron en España a través de una edición monográfica y excepcional de la revista cultural Debats ( ${ }^{\circ}$ 13, 1985, Valencia, Institució Alfons El Magnánim).

La respuesta de los teóricos españoles fue tardía y llegó de ámbitos académicos y profesionales diversos: historiadores, geógrafos, arquitectos e ingenieros. Desde la geografía llegaron las principales reacciones sobre la dimensión territorial de este patrimonio: Capel (1996) sostiene que los edificios industriales del pasado pueden ofrecer valores arquitectónicos que aconsejan su conservación y, a veces, su reutilización al servicio de operaciones más profundas de regeneración urbana, y pone de ejemplo la ciudad de Barcelona. Por su parte, Ortega (1998) afirma que «los territorios industriales sin uso constituyen el patrimonio industrial y forman, por ello, parte del patrimonio cultural». Otros geógrafos se muestran interesados por las oportunidades urbanísticas de los suelos industriales abandonados en grandes ciudades como Madrid (Pardo y Olivera, 1992); o bien profundizan en la relación entre patrimonio industrial y desarrollo local (Rodríguez, 1992; Troitiño, 1998), o entre patrimonio cultural y ordenación del territorio (Bielza y De Miguel, 1997). También los geógrafos 
se interesan por el papel que puede desempeñar el patrimonio industrial en la reactivación de espacios en crisis (López, 1992; Benito, 1997).

Asimismo, el interés de la sociedad española por las ruinas industriales se refleja en las reuniones y en los foros científicos. A la extensa bibliografía que se fue generando en la década de 1990 hay que sumar las ponencias y comunicaciones presentadas a congresos y encuentros nacionales e internacionales dedicados al patrimonio industrial. El debate teórico alcanzó profundidad y amplitud geográfica a partir del VII Congreso Internacional para la Conservación del Patrimonio Industrial, organizado en 1992 en Madrid por The Internacional Committee for the Conservation of the Industrial Heritage (TICCIH). Este organismo, creado en 1972 en el Reino Unido, impulsó el encuentro de expertos, la discusión teórica y el examen de las actuaciones sobre el patrimonio industrial en Europa y Latinoamérica; y sigue siendo un instrumento generador de debates y propuestas, como lo atestigua el último encuentro organizado en 2009 sobre Patrimonio Industrial y Obra Pública (TICCIH-España, 2010) y la exposición sobre Los 100 mejores edificios industriales en España (itinerante a lo largo de 2011).

En el ámbito internacional, organismos prestigiosos como la Unesco y el Icomos dan cobertura a la herencia industrial y favorecen su proyección social, declarando sitios industriales «Patrimonio de la Humanidad» y desplegando acciones de protección y conservación de edificios abandonados ligados a la memoria de las ciudades (Icomos, 1999). A su vez, este interés de las instituciones mundiales refuerza el interés local por el patrimonio industrial, lo que explica algunas acciones de notable alcance en ciudades medias y áreas rurales y mineras, donde se crean museos y centros de interpretación de la industria y se aprueban proyectos singulares sobre espacios abandonados (Incuna, 2002). En definitiva, a finales de la década de 1990, en toda Europa existe una evidente sensibilidad y conciencia social sobre la importancia y el valor de las ruinas industriales, pero prima la visión del monumento (el elemento aislado y singular) sobre lo que debería ser la incorporación estratégica de este recurso patrimonial a las políticas de desarrollo territorial y urbano.

\section{Programas de conservación y recuperación del patrimonio industrial}

En el seno de la Europa comunitaria, la sensibilidad hacia el patrimonio industrial se materializó en 1983 con la entrada en vigor del programa Apoyo a proyectos piloto comunitarios en materia de conservación del patrimonio arquitectónico, un instrumento financiero destinado a la conservación del patrimonio europeo de bienes inmuebles. Aunque importante, los resultados de este programa, operativo en el período 1986-1994, fueron insuficientes desde la óptica de la protección y conservación del patrimonio cultural en general, al fallar, sobre todo, los mecanismos de financiación (pocos proyectos subvencionados y recursos escasos para los que recibieron ayuda). Por lo que aquí interesa, hay que destacar que, de un total de 37 proyectos aprobados, 15 estuvieron relacionados directamente con la conservación y la rehabilitación de edificios 
y conjuntos fabriles repartidos entre Francia, Gran Bretaña, Alemania, Bélgica, Grecia, Dinamarca y España. Una modesta intervención que tiene, sin embargo, el valor simbólico de catalogar los vestigios industriales como elementos del patrimonio cultural, lo que les otorga un nuevo estatus (Benito, 2004).

Los fondos estructurales europeos, a través de diferentes programas comunitarios, también permitieron conservar y rehabilitar el patrimonio industrial. En el caso de regiones en declive, el Programa Nacional de Interés Comunitario y el RESIDER I hicieron posibles algunos proyectos como el Museo de la Minería del Entrego, la Ciudad Industrial de Valnalón, el Centro de Empresas Cristasa o el Centro de Empresas de La Curtidora, todos ellos localizados en el norte de España, en zonas mineras y siderúrgicas en crisis.

Con posterioridad, el Programa Cultura 2000, que reúne los antiguos programas europeos Raphael, Caleidoscopio y Ariane, se ocupó, en el período 20002004, del patrimonio cultural europeo y también de la herencia industrial. Esta iniciativa se amplió dos años más, 2004-2006, con una partida global de 190 millones de euros aplicados a financiar 1.294 acciones culturales, de las cuales 12 (un 0,93\%) están relacionadas con el patrimonio minero-industrial. Una modesta intervención que atestigua dos hechos importantes: primero, que en Europa se avanzó lentamente en la consideración social del patrimonio industrial, y, segundo, que dicho patrimonio se interpreta, en el siglo XXI, como un elemento de la cultura europea y se integra en las estrategias comunitarias, sobre todo en relación con las nuevas formas de turismo (Hidalgo, 2010).

A escala nacional, España puso en marcha, en el año 2000, el Plan Nacional de Patrimonio Industrial, uno de los más ambiciosos instrumentos europeos en la materia. Esta iniciativa se justifica por el valor testimonial de los restos industriales, considerados parte de la historia del país. El objetivo principal es utilizar el patrimonio industrial como factor de desarrollo económico y social para los territorios, para los lugares donde la industria fue importante y dejó su huella material e inmaterial (Hidalgo, 2010; Pardo, 2010). El ámbito de aplicación del plan es todo el territorio español, pero la intervención se realiza por fases y no es exhaustiva: primero, hay una selección de los bienes más valiosos a partir de un inventario general; segundo, se elabora un catálogo con los elementos seleccionados; tercero, se definen las actuaciones para proteger los bienes seleccionados, y, por último, se elabora un plan director para intervenir en su recuperación y puesta en valor. Hasta el momento, hay seleccionados 49 elementos, que están repartidos por la mayoría de las regiones españolas (se puede encontrar el listado completo en www.mcu.es/patrimonio/MC/IPHE/ PlanesNac/PlanIndustrial/PatrimonioIndustrial), y si bien el esfuerzo teórico está siendo importante, en la práctica no hay resultados palpables, es decir, la ejecución del plan no ha impulsado el deseado desarrollo territorial.

En definitiva, lo importante de los instrumentos y de las acciones descritas, más allá de su dimensión institucional y política, es verificar cómo se articulan en el territorio y hasta qué punto son capaces de generar prosperidad y bienestar en lugares donde la industria ha desaparecido y ha dejado tras de sí un panorama de atonía y crisis. Por el momento, los deseados efectos positivos 
son limitados y de intensidad débil. Muchas ciudades y regiones se hallan en la fase de intervención selectiva y tentativa, es decir, en la etapa dominada por acciones de rehabilitación de edificios catalogados y reutilización de suelos abandonados para desarrollos urbanos alternativos que aún no han producido sinergias de crecimiento apreciables.

Con carácter excepcional, algunos territorios pioneros aportan experiencias consolidadas que han resultado exitosas y son ejemplo de buenas prácticas en la recuperación del patrimonio industrial y su uso alternativo. Pensemos en la puesta en valor de antiguas minas, de viejas fábricas siderúrgicas o de colonias textiles en escenarios tan diversos como Manchester (Reino Unido), Valle del Rin (Alemania), Valle del Llobregat (Barcelona, España) o en la región Nord-Pas de Calais (Francia). Todos estos casos demuestran que es posible que la población y las instituciones se impliquen y colaboren de forma activa en el proceso de utilizar el patrimonio minero e industrial como un recurso, como un elemento generador de oportunidades. Y cuando esto sucede, se crea actividad y empleo, es decir, se dinamiza el territorio y las ciudades muestran la cara más positiva de su herencia industrial (véase $A V$ Monographs, ${ }^{\circ} 98$, 2002: New Old Europe).

\section{Paisajes e itinerarios industriales: nuevos enfoques integradores}

Hacia el año 2010, surgen propuestas creativas y originales para superar las acciones aisladas sobre los bienes y los espacios industriales abandonados. En este momento, en toda Europa, los gobiernos locales y regionales desarrollan, con desigual fortuna, instrumentos específicos para actuar en el patrimonio industrial con una perspectiva estratégica: recuperar áreas urbanas degradadas, dinamizar espacios mineros o impulsar el progreso en zonas rurales. Esto significa que la recuperación del patrimonio ya no se plantea a escala de edificio (objeto): no se trata de sembrar el territorio de monumentos industriales, aunque algunas fábricas tengan esta consideración.

Por otra parte, los museos de la industria también agotan su propuesta más convencional (edificios singulares con una colección de elementos técnicos y documentales sobre una actividad industrial y su historia encerrados en vitrinas y paneles) y se decide replantearlos como elementos o piezas de una red de museos que propone miradas cruzadas sobre actividades industriales coetáneas. Quizá el ejemplo más destacado sea Cataluña, donde se ha creado, en torno al Museo de la Ciencia y la Técnica de Tarrasa, todo un sistema descentralizado con 21 museos temáticos diferentes asociados en una red de amplia cobertura territorial y difusión en Internet. A esta iniciativa se suman los llamados museos del territorio o ecomuseos, una fórmula de éxito en Francia y en países centroeuropeos, pero menos extendida en España e Italia, que explican la industrialización de los lugares y permiten recuperar la memoria del trabajo y de la técnica con una gestión cultural avanzada (Pardo, 2010).

Otro hito en la nueva dimensión de las políticas de patrimonio industrial lo representa la idea de apostar por los itinerarios de la industrialización, por inter- 
venir en espacios complejos que son un compendio de factores que explican la industrialización histórica e, incluso, explican las causas de la desaparición de la industria tradicional; en suma, los itinerarios culturales que narran la historia del proceso de industrialización de los lugares se afianza como una alternativa a la intervención aislada y descontextualizada. Son proyectos que abarcan un amplio territorio, como es el caso del Parque Fluvial del Llobregat, que reúne el complejo entramado de las colonias textiles del siglo xIX repartidas por el río Llobregat, en las inmediaciones de Barcelona, donde su gran puerto comercial facilitó el desarrollo industrial de toda la comarca (Sabaté y Benito, 2010).

¿Qué finalidad tienen las nuevas intervenciones? ¿Qué papel desempeña el turismo en este proceso? Responder a estas cuestiones exige una mirada crítica sobre los espacios urbanos, las grandes ciudades y las zonas rurales y mineras donde la industria ha creado paisajes y ha absorbido terrenos hoy muy valiosos en términos económicos y urbanísticos. Los viejos edificios fabriles, las infraestructuras de transporte y comunicaciones y los suelos abandonados representan un recurso material, pues tienen un valor en el mercado inmobiliario y en el mercado del suelo, pero también representan una oportunidad, ya que sirven para crear productos culturales de interés turístico en el marco de sociedades avanzadas que reclaman de manera creciente inversiones en actividades de ocio y recreo.

El turismo industrial se afianza en todas las regiones de antigua industrialización de Europa como una actividad alternativa y pujante. Esto exige la reordenación de los espacios abandonados y la intervención planificada sobre el patrimonio industrial. Como hemos visto, se han recuperado bienes industriales para usos alternativos y que mejoran los entornos; se han creado redes de museos temáticos y centros de interpretación de la industria; se está trabajando en itinerarios que comprenden áreas urbanas, paisajes naturales, infraestructuras ferroviarias, obras de ingeniería, poblados obreros, etcétera; todo para componer un producto turístico capaz de crear empleo y riqueza en lugares desfavorecidos. Esta manera de actuar es lícita, sin duda, pero tiene un riesgo: la banalización del territorio y su memoria, pues los parques industriales pueden derivar en negocios efímeros que sacrifican una herencia muy valiosa para la población y la memoria del lugar. Por tanto, el turismo industrial debe responder a un proyecto racional y meditado sobre los recursos disponibles y la capacidad de los territorios para encajar una nueva actividad que debe, a la vez, proteger, conservar y poner en valor, evitando riesgos de destrucción o degradación del patrimonio por un uso abusivo o inadecuado del mismo (Icomos, 1999; Ticcih, 2003).

Asimismo, los elementos y los bienes vinculados a las actividades industriales cobran protagonismo en las políticas de patrimonio cultural: bien porque se trata de una herencia valiosa; bien porque forman parte de paisajes urbanos o rurales. Según el Convenio Europeo del Paisaje, aprobado en Florencia el año 2000, dichos paisajes deben integrarse en las estrategias de desarrollo territorial, al mismo tiempo que forman parte de la defensa de la cultura mundial. Además, el uso turístico del patrimonio requiere una negociación entre los 
agentes políticos y sociales fundada en una conciencia clara de su valor como recurso y como elemento identitario (Harrison y Hitchcock, 2005; Graham et al., 2000).

En consecuencia, los gobiernos se afanan por registrar su patrimonio, por elaborar los llamados inventarios de patrimonio industrial histórico como paso previo para elaborar políticas de puesta en valor y uso competitivo. Dichos inventarios, realizados en Francia, Alemania, Reino Unido, Italia y España, entre otros países, y perfeccionados metodológicamente a lo largo de dos décadas de experiencia, son un instrumento muy útil en la toma de decisiones. Realmente, son una herramienta necesaria para las políticas de ordenación del territorio y de planificación, pues identifican paisajes y construcciones que hay que proteger porque son vulnerables o porque son valiosos y pueden actuar como foco turístico y recurso cultural (Conséil de l'Europe, 2002; Cañizares, 2010). Pero los paisajes industriales precisan, antes de su explotación turística, un análisis responsable por parte de los gestores públicos, lo que implica, al menos, tres tareas que competen a los gobiernos (Zoido, 2010):

- Formular los objetivos de calidad paisajística para todo el territorio nacional.

- Asumir responsabilidades en la protección, gestión y ordenación de los paisajes transfronterizos.

- Incluir y desarrollar la dimensión paisajística en las políticas sectoriales.

Solo actuando de este modo se garantiza una intervención responsable, ordenada y de éxito en los paisajes que se transforman en producto turístico. Los planificadores deben proyectar infraestructuras nuevas y centros de recepción de visitantes, a los que hay que ofrecer alojamientos y medios de movilidad. Esto implica a los agentes económicos y sociales, que tienen que invertir en crear esos recursos y gestionar su funcionamiento. Todo un reto para los territorios que apuestan por utilizar su patrimonio con fines productivos y un desafío para la sociedad, que debe buscar el equilibrio entre desarrollo y sostenibilidad.

\section{Patrimonio industrial, identidad y proyecto local: planteamientos a debate}

La renovación en la percepción y las propuestas de uso del patrimonio industrial llega de la mano de aquellos autores que plantean una lectura subjetiva de los paisajes, de todo tipo de paisajes: los paisajes materiales y concretos, resultado de procesos complejos en el tiempo debido a la reiterada acción del hombre sobre el medio; y los paisajes producto de la memoria y de la percepción. Se interpreta el paisaje como una construcción social y se concibe como «extensión del patrimonio cultural y de su interpretación» (Cruz y Español, 2009; Nogué, 2007).

Este discurso incluye a «los otros paisajes», aquellos que están al margen y que no vemos por razones diversas, pero que demuestran que el paisaje es, en 
efecto, una construcción social (Nogué, 2007, 2008). Según este autor, los paisajes son el reflejo de la organización del territorio y se construyen socialmente en el marco de complejas y cambiantes relaciones de clase, de género, de etnia y de poder. Por eso mismo, las miradas sobre los paisajes son diversas y, a veces, contrapuestas, pero todas ellas imprescindibles para comprender la relación del ser humano con el territorio, con los lugares. La importancia creciente de lo intangible queda demostrada por la lista de los 46 elementos declarados por la Unesco Patrimonio Cultural Inmaterial de la Humanidad, entre los que cabe citar el carnaval belga de Aalst, la ópera de Pekín, el flamenco español, el sistema normativo de los wayuu de Colombia o las técnicas artesanales tradicionales de tejido de alfombras en Kashan (Irán) (véase http://www.unesco. $\mathrm{org} / \mathrm{new} / \mathrm{es} / \mathrm{media-services/single-view/news/).} \mathrm{No} \mathrm{solo} \mathrm{importan} \mathrm{los} \mathrm{sitios,}$ evidentemente tangibles, sino también las expresiones del pensamiento, los sentimientos colectivos y ciertas formas de vida que tienen su proyección, sin duda, en los paisajes.

Volviendo sobre la percepción de los paisajes, entre los que no encajan en la idea convencional de paisaje se hallan las ruinas industriales, las viejas fábricas abandonadas que siguen hoy provocando rechazo e incomprensión. Son, para muchos, elementos obsoletos, testimonio de un fracaso que se quiere olvidar y borrar de la memoria. O lo que es equivalente: los paisajes que se desea transformar para crear algo nuevo y diferente haciendo tabla rasa del pasado. Con frecuencia, las intervenciones en el patrimonio industrial van en esta línea: crear a partir de un viejo contenedor un nuevo edificio, una construcción diferente y moderna que nos devuelva una imagen positiva y de progreso. También hay intervenciones que arrasan los viejos edificios y estructuras industriales para obtener suelo para usos residenciales y terrenos para nuevas actividades económicas.

Eliminar ciertos elementos del paisaje, o directamente sustituir un paisaje por otro, tiene relación con un componente hasta ahora poco sometido a consideración: la identidad. Y en relación con ella, la idea de proyecto local. Según destaca Conti (2011), la valorización de las especificidades territoriales genera ventajas competitivas localizadas que permitirían a los distintos lugares formar parte de las redes globales y beneficiarse así de tendencias de crecimiento y expansión ligadas a procesos originados en múltiples sitios a la vez. En nuestro caso, esto supone reformular el concepto de patrimonio como proyecto local. Bajo esta perspectiva, el patrimonio industrial se inserta en un contexto más amplio: la dimensión local del desarrollo, que implica superar la idea del territorio como soporte de recursos susceptibles de ser explotados en favor de una visión del territorio como patrimonio a valorizar y con elementos de extraordinario interés y potencial (Magnaghi, 2000).

En esta interpretación, el patrimonio industrial es una seña de identidad de los lugares, un elemento distintivo que permite articular proyectos singulares apoyados por la población y los agentes que intervienen en la producción y la gestión de espacios culturales (Dewarrat, 2003; Graham y Howard, 2009). La población se identifica con la herencia industrial del lugar (memoria del 
trabajo, memoria de la vida cotidiana, memoria social) y se involucra en dichos proyectos, participando de las propuestas institucionales y cooperando en las iniciativas de recuperación y puesta en valor de dicho patrimonio, representado por bienes aislados, de carácter monumental, y por paisajes antaño despreciados, olvidados o considerados kitsch (Atkinson, 2007).

Por último, hay planteamientos que reivindican el valor de las ruinas como generadoras de un paisaje genuino, en el que no hay que intervenir para cambiar su apariencia. Se trata de una defensa de lo bruto, lo feo, los restos de una actividad agresiva y abandonada que deja profundas cicatrices en el paisaje, sobre todo en el paisaje urbano. Estas ruinas se sitúan, según el británico Edensor (2005a), en el centro de la memoria social individual y cobran nuevo sentido. También adquieren un enorme potencial como elemento transgresor y provocativo: frente a los cuidados y ordenados paisajes que se prodigan en las ciudades occidentales, los espacios industriales abandonados son un reto para los políticos, los urbanistas y los propios ciudadanos, ya que ofrecen una imagen fantasmal del pasado que obliga a enfrentarse a una materialidad incómoda. Son paisajes en peligro que están fuertemente arraigados en la memoria y el sentido del lugar, y que desafían a los sentidos, razones por las que Edensor (2005b) defiende su existencia en el marco de un urbanismo más flexible y lúdico.

\section{Conclusiones}

Es importante constatar y poner énfasis en el hecho de que los edificios y los recintos industriales abandonados son percibidos cada vez con más frecuencia como elementos del patrimonio cultural y no como una ruina inservible; como recursos o bienes activos con enormes posibilidades en el ámbito de la arquitectura, el urbanismo y el tratamiento de los paisajes en general. También la herencia industrial se contempla como un recurso en el que apoyar acciones de reactivación de la economía urbana o audaces intervenciones que cambian la imagen y la funcionalidad de las ciudades, tanto grandes como pequeñas.

Por su parte, el nuevo urbanismo encuentra en las viejas fábricas el pretexto perfecto para generar espacios de nueva centralidad y transformar edificios abandonados en contenedores con usos que van desde auditorios y galerías de arte hasta centros comerciales o apartamentos de viviendas, todo ello aderezado con arquitecturas que reinterpretan lo viejo con lenguajes actuales. Los terrenos industriales abandonados son, a su vez, escenario y soporte de proyectos que aúnan el negocio con operaciones de expansión y regeneración de barrios degradados. En este sentido, parece que el patrimonio industrial ha encontrado su lugar en la ciudad, su sentido como elemento a respetar e integrar en el planeamiento urbanístico y en los proyectos de cirugía urbana, así como en su condición de contenedor de usos alternativos de calidad ligados a los negocios, la cultura y el arte.

También en relación con el turismo se afianza este tipo de patrimonio: fábricas museo, museos de objetos y artefactos industriales, museos sobre la his- 
toria del trabajo y la técnica, museos territoriales, etcétera. Una oferta variada que puede resultar reiterativa y redundante cuando varios museos de temática similar se multiplican en un radio limitado. Un asunto que bien merece someterse a debate, pues está generando problemas de saturación en determinados lugares que compiten por atraer visitas y recaudar ingresos.

En materia de patrimonio industrial se debe prestar atención, asimismo, al conjunto de elementos patrimoniales que crean paisajes únicos y singulares. No se trata tanto de actuar e intervenir en elementos aislados, de gran presencia y obvia reutilización, cuanto de actuar en paisajes urbanos y rurales donde la huella de la industria forma parte del espacio geográfico, de la cultura del territorio.

El reto en los próximos años no radica en seguir abriendo museos y perseverar en el tipo de acciones e intervenciones antes descritas, muchas oportunas y loables, y sin duda un verdadero avance. Hay que ir más allá del monumento y pensar en el territorio; superar la perspectiva del hecho industrial - expresado en el edificio- $-y$ pensar en el proceso de industrialización de los lugares. Las acciones de recuperación y puesta en valor del patrimonio industrial deben cambiar, además del enfoque, de escala: pasar del elemento o edificio al paisaje. Los paisajes mineros, los paisajes siderúrgicos, los paisajes harineros, los paisajes portuarios..., todos ellos deberían recibir un tratamiento específico, pero de conjunto. Y ser objeto de propuestas integrales que vinculen las arquitecturas con los demás elementos del entorno.

En consecuencia, parece conveniente evolucionar del objeto monumento al paisaje itinerario: enlazar de forma coherente arquitecturas industriales dispersas y crear circuitos que expliquen la industrialización del territorio. Esto implica prestar atención a todos los factores que han hecho posible una industria y la organización del espacio que su presencia conlleva: desde la existencia de materias primas y fuentes de energía, pasando por los sistemas de transportes y distribución de mercancías, hasta las cuestiones relacionadas con la localización y la construcción de las fábricas. Un circuito productivo que implica a la población y a sus formas de asentamiento (pueblos, villas, ciudades) y que ha creado paisajes realmente singulares y con fuerte carga patrimonial y cultural, en espacios litorales y de interior; en valles de montaña $\mathrm{y}$ en mesetas y deltas.

Muchos de estos paisajes están en peligro porque no se reconoce su valor cultural y se actúa aisladamente en sus elementos, con lo cual se rompe el discurso que da unidad al conjunto y lo explica. No es razonable, ni sostenible, actuar sobre elementos aislados, por muy valiosos que sean, ignorando el paisaje creado. En este momento, hay madurez profesional y social como para defender un proyecto unitario e integrador que bien puede desarrollarse por fases, sin perder la perspectiva de rescatar un complejo espacial sujeto a la lógica de la industrialización. Esta sería mi propuesta y el reto mayor que atisbo en el horizonte del patrimonio y la arquitectura industrial. 


\section{Bibliografía}

Actas VII Congreso Internacional para la Conservación del Patrimonio Industrial (1995). Madrid: TICCIH- CEHOPU.

Actas IX Conferencia Internacional sobre Conservación de Centros Históricos y del Patrimonio Edificado (1997). Valladolid: Instituto de Urbanística de la Universidad de Valladolid.

Álvarez, M.A. (1992). «Recuperación y uso del patrimonio industrial». ABACO Revista de Ciencias Sociales, 1 (2), 57-66.

- (2001). «Patrimonio industrial, identidad cultural y sostenibilidad». En: Arqueología industrial, patrimonio y turismo cultural Gijón: Incuba, 13-32.

Atkinson, D. (2007). «Kitsch Geographies and the Everyday Sapaces of Social Memory». Environment and Planning A, 39, 421-440.

Bardon, A. (2001). "Una memoria oxidada». Fuentes [en línea], 131 <www.fuentesunesco.org>.

Benito, P. (1993). "El problema de las ruinas industriales en Europa». Boletín de Información sobre las Comunidades Europeas. Oviedo: Universidad de Oviedo y Principado de Asturias, 22-26.

- (1997). «Dinamización del territorio y patrimonio industrial». Polígonos, 7, 123-131.

- (2004). «Patrimonio industrial y cultura del territorio». Boletín de la Asociación de Geógrafos Españoles, 34, 213-228.

Bergerón, L. y Dorel-Ferre, L. (1996). Le Patrimoine Industriel: Un nouveau territoire. París: Éditions Liris.

Bergerón, L. (2002). «El patrimonio industrial, ¿qué hacer?». En: Álvarez, M.A. (coord.). Patrimonio industrial: Lugares de la memoria. Gijón: Incuna, 11-16.

Bielza, V. y Miguel, R. de (1997). "El patrimonio cultural, componente de la ordenación del territorio». Revista Aragonesa de Administración Pública, 10, 185-195.

Borsi, F. (1978). Introduzione alla archeología industriale. Roma: Offizina Edizione.

Buchanan, R.A. (1972). Industrial Archaeology in Britain. Londres: Penguin Books.

Cañizares, Ma C. (2010). "El patrimonio industrial: un recurso del territorio y un componente del paisaje». En: V Congreso TICCIH-España: Patrimonio Industrial y Paisaje. Gijón: TICCIH, 497-506.

Capel, H. (1996). "La rehabilitación y el uso del patrimonio histórico industrial». Documents d'Anàlisi Geogràfíca, 29, 19-50.

Conseil de l'Europe (2002). Fonctions du patrimoine culturel dans une Europe en changement. Estrasburgo: UE Press.

Conti, S. (2011). «Recualificación y uso de los suelos industriales abandonados». En: Benito, P. (dir.). Areas empresariales, suelo industrial y logística: Análisis y procesos en el territorio. Navarra: Thomson Reuters, 15-32.

Cossons, N. (1975). The BP book of the Industrial Archaeology. Newton Abbot: D. \& Ch. Publishers.

Cruz, L. y Español, I. (2009). El paisaje: De la percepción a la gestión. Madrid: Liteam. Daumas, M. (1980). L'Arqueologie industrielle en France. París: Ed. Robert Laffont.

DEBATS (1985). "La arqueología industrial». Debats, 13. Valencia: Institució Alfons el Magnànim.

Dewarrat, J-P et al. (2003). Paysages ordinaires: De la protection au proyect. Sprimont: Pierre Mardaga.

EDEnsor, T. (2005a). "The ghosts of industrial ruins: ordering and disordering memory in excessive space». Environment and Planning D: Society and Space, 23, 829-849. 
- (2005b). Industrial Ruins: Space, Aesthetics and Materiality. Oxford: Berg.

FELIú, A. (1998). "El patrimonio industrial, localizaciones, regeneraciones: una nueva geografía». Ábaco: Revista de Cultura y Ciencias Sociales, 19, 71-80.

FERBER, U. (1994). «Rehabilitación, ocio y turismo: ¿Qué perspectivas presentan para la gestión de las áreas industriales abandonadas». En: Territorios de Europa: La problemática de los espacios industriales abandonados. Valladolid: Junta de Castilla y León, 65-74.

Forner, S. (1989). «Dossier arqueología industrial». Canelobre, 16, 17-76.

Graham, B. et al. (2000). A Geography of Heritage: Power, Culture and Economy. Londres: Arnold.

Graham, B. y Howard, P. (2009). Heritage and Identity. Ashgate Research Companion.

Harrison, D. y Hitchсоск, M. (2005). The Politics of World Heritage: Negotiating, Tourism and Conservation. Cleveden: Channel View Publication.

Hidalgo, C. (2010). El patrimonio minero-industrial y ferroviario: nuevos recursos para nuevos turismos. Madrid: Universidad Autónoma de Madrid.

Hudson, K. (1963). Industrial Archaeology. An Introduction. Londres: John Baker.

- (1979). World Industrial Arqueology. Cambridge: Cambridge University Press.

ICOMOS (1999). Carta Internacional sobre Turismo Cultural [en línea]. México. $<$ www.international.icomos.org $>$.

INCUNA (2002). Patrimonio Industrial: Lugares de la memoria. Proyectos de reutilización en industrias culturales, turismo y museos. Gijón: CICEES.

Lista, A. y Sabaté, J. (2001). Projectant l'eix del Llobregat: Paisatge cultural i desenvolupament regional. Barcelona: UPC-MIT.

López, M. (1992). «El concepto de patrimonio. El patrimonio industrial o la memoria del lugar». Ábaco: Revista de Cultura y Ciencias Sociales, 1, 9-12.

Lorimer, H. (2005). "Cultural geography: the business of being "more than representational"”. Progress in Human Geography, 29, 83-94.

Maderuelo, J. (dir.) (2010). Paisaje y patrimonio. Madrid: ABADA Editores.

Magnaghi, A. (2000). Il progetto locale. Turín: Bollati Boringhieri.

Mitchell, D. (2003). "Cultural landscapes: just landscapes or landscapes of justice?». Progress in Human Geography, 27, 787-796.

Nogué, J. (ed.) (2007). La construcción social del paisaje. Madrid: Biblioteca Nueva.

- (2008): "Al margen: Los paisajes que no vemos». En: Maderuelo, J. Paisaje y territorio. Madrid: ABADA Editores, 181-202.

OrtegA, J. (1998). "El patrimonio territorial: El territorio como recurso cultural y económico». Ciudades, 4, 33-48.

PARDo, C. (2010). «El patrimonio industrial en España: análisis turístico y significado territorial de algunos proyectos de recuperación». Boletín de la Asociación de Geógrafos Españoles, 53, 239-266.

Pardo, C. y Olivera, A. (1992). «Les friches industrielles à Madrid: Opportunités urbanistiques et réutilisation du sol urbain». Revue Belge de Géographie, 116 (51), $1-4$.

Pinard, J. (1985). L'archéologie industrielle. París: Puf.

Programa Comunitario RECITE Universidades-Regiones (1994). La problemática de los espacios industriales degradados. Valladolid: Junta de Castilla y León/Cedre, vol. I.

Rodríguez, F. (1992). «El patrimonio industrial histórico como recurso para el desarrollo local». Ábaco: Revista de Cultura y Ciencias Sociales, 1, 71-78. 
Sabaté, J. y Benito, P. (2010). «Paisajes culturales y proyecto territorial: un balance de treinta años de experiencia». Identidades. Territorio, Proyecto, Patrimonio, 2, 7-26.

Schama, S. (1995). Landscape and Memory. New Cork: Vintage Books.

TICCIH (2003). Carta de Nizhny Tagil sobre Patrimonio Industrial [en línea]. <www. international.icomos.org $>$.

TICCIH-España (2010). Actas V Congreso Conservación del Patrimonio Industrial y de la Obra Pública en España. Gijón: Editorial CICEES.

Troitiño, M.A. (1998). "Patrimonio arquitectónico, cultura y territorio». Ciudades, 4, 95-104.

Zoido, F. (2010). «Territorio y paisaje, conocimiento, estrategias y políticas». En Pillet, F. et al. Territorio, paisaje y sostenibilidad. Barcelona: Ediciones del Serbal, 85-112. 